\title{
ON THE MARKOV-KREIN IDENTITY AND QUASI-INVARIANCE OF THE GAMMA PROCESS
}

\author{
A. Vershik, M. Yor, and N. Tsilevich
}

UDC 512

We present a simple proof of the Markov-Krein identity for distributions of means of linear functionals of the Dirichlet process and its various generalizations. The key idea is to use the representation of the Dirichlet process as the normalized gamma process and fundamental properties of gamma processes. Bibliography: 19 titles.

\section{Dedicated to the memory of S. V. Kerov}

1. Introduction: Dirichlet processes, gamma processes and the Markov-Krein identity. The purpose of this paper is to apply the presentation of Dirichlet processes as the normalized gamma processes to prove the Markov-Krein identity for distributions of means of Dirichlet processes. This approach turns out to be very fruitful; in particular, it allows us to obtain the desired formula almost immediately.

The Dirichlet processes introduced in [4] play a key role in Bayesian nonparametric statistics. The classical definition of these processes is the following. Denote by

$$
\Delta_{n}=\left\{x=\left(x_{0}, \ldots, x_{n}\right): x_{i} \geq 0, \sum x_{i}=1\right\}
$$

the standard $n$-dimensional simplex. The Dirichlet distribution $\operatorname{Dir}\left(\tau_{0}, \ldots, \tau_{n}\right)$ on $\Delta_{n}$ with parameters $\tau_{0}, \ldots$, $\tau_{n}>0$ is determined by the density

$$
\frac{\Gamma\left(\tau_{0}+\ldots+\tau_{n}\right)}{\Gamma\left(\tau_{0}\right) \ldots \Gamma\left(\tau_{n}\right)} x_{0}^{\tau_{0}-1} \ldots x_{n}^{\tau_{n}-1}
$$

with respect to the Lebesgue measure on $\Delta_{n}$.

Definition 1. Let $(X, \nu)$ be a standard Borel space with a nonatomic finite positive measure $\nu$. A Dirichlet process on the space $X$ with parameter measure $\nu$ is a random probability distribution $P$ on $X$ such that for every finite measurable partition $X=A_{0} \cup \ldots \cup A_{n}$ the vector $\left(P\left(A_{0}\right), \ldots, P\left(A_{n}\right)\right)$ has Dirichlet distribution $\operatorname{Dir}\left(\nu\left(A_{0}\right), \ldots, \nu\left(A_{n}\right)\right)$ on $\Delta_{n}$.

Denote by $\theta=\nu(X)$ the total charge of the measure $\nu$, and let $\bar{\nu}=\nu / \theta$ be the normalized measure $\nu$. An explicit construction of the Dirichlet process is given by the following formula:

$$
P=\sum_{i=1}^{\infty} Q_{i} \delta_{Y_{i}}
$$

where $Y=\left(Y_{1}, Y_{2}, \ldots\right)$ is a sequence of i.i.d. variables on the space $X$ with common distribution $\bar{\nu}$, and $Q=\left(Q_{1}, Q_{2}, \ldots\right)$ is a random point of the infinite-dimensional simplex

$$
\Sigma=\left\{x=\left(x_{1}, x_{2}, \ldots\right): x_{1} \geq x_{2} \geq \ldots \geq 0, \sum x_{i}=1\right\}
$$

that is independent of $Y$ and has the Poisson-Dirichlet distribution $P D(\theta)$ with parameter $\theta$.

Many papers are devoted to the study of the distributions of random means of Dirichlet processes. Let $a: X \rightarrow \mathbb{R}$ be a measurable function. It defines a linear functional $f_{a}(\xi)=\int_{X} a(x) d \xi(x)$ on the space of Borel measures on $X$. The problem is to describe the distribution $\mu_{a}$ of this functional with respect to the Dirichlet process $P$. The answer is as follows. Let $\nu_{a}$ be the distribution of the function $a$ with respect to the normalized parameter measure $\bar{\nu}$. Then the measures $\mu_{a}$ and $\nu_{a}$ are related by the following integral identity:

$$
\int_{\mathbb{R}} \frac{1}{(1+z u)^{\theta}} d \mu_{a}(u)=\exp \left(-\int_{X} \log (1+z u)^{\theta} d \nu_{a}(u)\right) .
$$

Translated from Zapiski Nauchnykh Seminarov POMI, Vol. 283, 2001, pp. 21-36. Original article submitted November $15,2001$. 
If the measures under consideration have finite moments of all orders, then identity $(*)$ is equivalent to the following moment identity (regarded as an equality of formal power series):

$$
\sum_{n=0}^{\infty} h_{n} z^{n}=\exp \sum_{n=1}^{\infty} \frac{p_{n}}{n} z^{n}
$$

where $h_{n}=\int u^{n} d \mu_{a}(u)$ and $p_{n}=\int u^{n} d \nu_{a}(u)$ are the moments of the measures $\mu_{a}$ and $\nu_{a}$ respectively. Note that this identity coincides with the identity from the theory of symmetric functions that relates complete symmetric functions and power sums (see [12]).

Formula $(*)$ was first obtained in [2] by hard analytic arguments (see also simpler proofs in [3, 8]). In the case $\theta=1$ this identity means that the distribution $\mu_{a}$ is the Markov-Krein transform of the measure $\nu_{a}$ (the term suggested by S. V. Kerov). This transform arises in many various contexts like the Markov moment problem, continued fractions theory, exponential representations of analytic functions, the spectral shift function of a self-adjoint operator, the Plancherel growth of Young diagrams, etc. (see Kerov [7] for a detailed survey). It first appeared in A. A. Markov's paper [13], and later was intensively studied by M. G. Krein and his school. In particular, this identity gives a link between the so-called Markov moment problem and the classical Hausdorff moment problem. Let us briefly discuss this link.

Recall that the Hausdorff moment problem consists in describing all sequences $s_{0}, s_{1}, \ldots$ that arise as the moment sequences of probability measures $\varkappa$ on $[0,1]$. The solution of this problem is well known. A less known Markov moment problem is to describe all sequences $m_{0}, m_{1}, \ldots$ that arise as the moment sequences of absolutely continuous measures $f(t) d t$ on $[0,1]$ with bounded densities: $0 \leq f(t) \leq 1$. It turns out (see [10]) that the second problem can be reduced to the first one as follows. There is a one-to-one correspondence between bounded densities $0 \leq f(t) \leq 1$ on $[0,1]$ and probability measures $\varkappa$ on $[0,1]$ given by the formula

$$
\int_{0}^{1} \frac{d \varkappa(t)}{z-t}=\frac{1}{z} \exp \int_{0}^{1} \frac{f(t) d t}{z-t} .
$$

In terms of moments, this identity takes the form

$$
\sum_{n=0}^{\infty} s_{n} z^{-n}=\exp \sum_{n=1}^{\infty} m_{n-1} z^{-n}
$$

This allows one to explicitly express the moments of $\varkappa$ via the moments of $f(t) d t$. It follows that $\left\{m_{n}\right\}$ is a Markov moment sequence if and only if the sequence $\left\{s_{n}\right\}$ constructed by the above formula is a Hausdorff moment sequence. There is an explicit probabilistic procedure due to S. Kerov [6] for constructing the measure $\varkappa$ related to a given measure $f(t) d t$ by $(* *)$. In order to reduce $(* *)$ to $(*)$, one should take $f(t)=\nu_{a}((t, \infty))=$ $\nu(\{x \in X: a(x)>t\}), \varkappa=\mu_{a}$, replace $z$ by $-\frac{1}{z}$ and integrate by parts.

In this note we present a new and remarkably simple proof of the Markov-Krein identity for the distribution of means of Dirichlet processes which is based on the close relation between Dirichlet processes and gamma processes. The starting point of our approach is the following obvious fact.

The link between Dirichlet processes and gamma processes. The Dirichlet process on the space $X$ with parameter measure $\nu$ is the normalized gamma process $\bar{\gamma}(x)=\gamma(x) / \gamma(X)$.

Thus, the problem of describing the distributions of means of Dirichlet processes can be formulated in terms of gamma processes as follows. Given a linear functional $f_{a}(\xi)=\int_{X} a(x) d \xi(x)$ determined by a function a $: X \rightarrow$ $\mathbb{R}_{+}$, describe the distribution $\mu_{a}$ of the functional $f_{a}$ with respect to the normalized gamma process in terms of the distribution $\nu_{a}$ of the function $a$.

This reduction to gamma processes is a powerful tool for the study of Dirichlet processes. In particular, even the simplest properties of the gamma processes make it possible to easily obtain formula $(*)$. The crucial point is the following fundamental property.

Independence property. The normalized gamma process $\bar{\gamma}$ and the total charge $\gamma(X)$ are independent.

According to a personal communication of S. Kerov, in the simplest case of discrete parameter measure $\nu$ (in this case the gamma process is simply a sum of independent gamma variables, and the normalized gamma process is a random point of a finite-dimensional simplex with Dirichlet distribution), the idea of proving (*) via 
the Laplace transform of gamma variables was used by F. Huffer. However, it was not noticed earlier that the general case of arbitrary parameter measures can be handled in a strikingly simple way by using general gamma processes.

The independence property leads to many other distinguished properties of gamma processes, e.g., the socalled multiplicative quasi-invariance, see [17, 19].

Quasi-invariance property. The gamma process $\gamma$ on the space $(X, \nu)$ is quasi-invariant with respect to multiplicators $M_{a} \gamma(x)=a(x) \gamma(x)$ for all functions

$$
a: X \rightarrow \mathbb{R}_{+} \text {with } \int_{X}|\log a(x)| d \nu(x)<\infty
$$

We would also like to mention that the law of the gamma process admits an equivalent sigma-finite measure which is already invariant with respect to all multiplicators $M_{a}$. This measure enjoys many important properties; it is a natural infinite-dimensional analogue of the Lebesgue measure, and plays a key role in the representation theory of the current group $S L(2, \mathbb{R})^{X}$. See [19] for a detailed treatment of this topic.

We would like to emphasize that the transition from Dirichlet processes to gamma processes (i.e., from the normalized process to the nonnormalized one, or from the simplex to the cone) makes the proof of the MarkovKrein formula $(*)$ for Dirichlet processes remarkably simple. Thus it would probably be fruitful to consider the Markov moment problem itself, and numerous related questions, from this standpoint. It is interesting that a very similar lifting from the simplex to the cone was recently used by A. Borodin and G. Olshanski in their work on the harmonic analysis on the infinite symmetric group and point processes, see, e.g., [1].

The Markov-Krein identity (*) may also be interpreted as a relation between the Laplace transform of the distribution of the functional $f_{a}$ with respect to the gamma process and the Cauchy transform of the distribution of the same functional with respect to the normalized gamma process.

Our interpretation of the Markov-Krein identity leads to the following general problem: given an arbitrary Lévy process $\eta$ on $(X, \nu)$ and a function $a: X \rightarrow \mathbb{R}_{+}$, describe the relation between the distribution of the functional $f_{a}$ with respect to $\eta$ and the distribution of the same functional with respect to the normalized Lévy process $\bar{\eta}$.

A preliminary exposition of the main results of this paper appeared as a preprint [18].

2. General Lévy processes. It is natural to consider Dirichlet processes in the context of general Lévy processes. In this section we present the necessary background following $[17,19]$.

Let $(X, \nu)$ be a standard Borel space with nonatomic finite nonnegative measure $\nu$, and let $\nu(X)=\theta$ be the total charge of $\nu$. Denote by

$$
D=\left\{\sum z_{i} \delta_{x_{i}}, x_{i} \in X, z_{i} \in \mathbb{R}, \sum\left|z_{i}\right|<\infty\right\}
$$

the real linear space of all finite real discrete measures on $X$, and let $D^{+}=\left\{\sum z_{i} \delta_{x_{i}} \in D: z_{i}>0\right\} \subset D$ be the cone in $D$ consisting of all positive measures.

Let $\Lambda$ be a measure on $\mathbb{R}_{+}$that satisfies the following conditions:

$$
\begin{array}{ll}
\Lambda(0, \infty)=\infty, & \Lambda(1, \infty)<\infty, \\
\int_{0}^{1} s d \Lambda(s)<\infty, & \Lambda(\{0\})=0 .
\end{array}
$$

Denote by $\psi_{\Lambda}$ the Laplace transform of the infinitely divisible distribution $F_{\Lambda}$ with Lévy measure $\Lambda$ :

$$
\psi_{\Lambda}(t)=\exp \left(-\int_{0}^{\infty}\left(1-e^{-t s}\right) d \Lambda(s)\right)
$$

Each bounded Borel function $a: X \rightarrow \mathbb{R}$ defines a linear functional $f_{a}$ on $D$ by the formula $f_{a}(\eta)=$ $\int_{X} a(x) d \eta(x), \eta \in D$. 
Definition 2. A Lévy process on the space $(X, \nu)$ with Lévy measure $\Lambda$ satisfying (2) is a generalized process on $D$ whose law $P_{\Lambda}$ has Laplace transform

$$
\mathbb{E}\left[\exp \left(-\int_{X} a(x) d \eta(x)\right)\right]=\exp \left(\int_{X} \log \psi_{\Lambda}(a(x)) d \nu(x)\right),
$$

where $a$ is an arbitrary nonnegative bounded Borel function on $X$.

It is easy to show that the distribution $P_{\Lambda}$ of a Lévy process is supported by the cone $D^{+}$of positive discrete measures.

Consider the cone $C=\left\{z=\left(z_{1}, z_{2}, \ldots\right): z_{1} \geq z_{2} \geq \ldots \geq 0, \sum z_{i}<\infty\right\} \subset l^{1}$ and define a map $T: D^{+} \rightarrow$ $C \times X^{\infty}$ by the formula

$$
T \eta=\left(\left(Q_{1}, Q_{2}, \ldots\right),\left(X_{1}, X_{2}, \ldots\right)\right) \quad \text { if } \quad \eta=\sum Q_{i} \delta_{X_{i}} .
$$

Definition 3. Let $P$ be a distribution on the space $D^{+}$, and let $\eta=\sum Q_{i} \delta_{X_{i}}$ be a random process obeying $P$. The random sequence of charges $\left(Q_{1}, Q_{2}, \ldots\right)$ is called the conic part of the process $\eta$, and its distribution on the cone $C$ is called the conic part of the law $P$.

It is not difficult to show that the conic part of the Lévy process with Lévy measure $\Lambda$ is the ordered sequence of points of the Poisson process on $\mathbb{R}_{+}$with mean measure $|\nu| \Lambda$. Thus the conic part depends only on the Lévy measure $\Lambda$ and on the full charge of the parameter measure $\nu$. The following theorem shows that the study of a Lévy process can be essentially reduced to the study of its conic part since the construction of the process involves the parameter measure in a trivial way. This fundamental property of Lévy processes is a particular case of the theorem proved first in [5]. A simpler proof of this fact is given in [18].

Theorem 1 ([5, 18]). Let $\eta=\sum Q_{i} \delta_{X_{i}}$ be a Lévy process on the space $(X, \nu)$ with Lévy measure $\Lambda$. Then $T P_{\Lambda}=\varkappa_{|\nu| \Lambda} \times \bar{\nu}^{\infty}$, i.e., $X_{1}, X_{2}, \ldots$ is a sequence of i.i.d. random variables with common distribution $\bar{\nu}=\nu /|\nu|$, and this sequence is independent of the conic part $\left(Q_{1}, Q_{2}, \ldots\right)$.

Denote by $D_{1}^{+} \subset D^{+}$the simplex of all normalized atomic measures. Then $D^{+}=D_{1}^{+} \times[0, \infty)$, i.e., each $\eta \in D^{+}$can be represented as

$$
\eta=(\eta / \eta(X), \eta(X))
$$

where $\eta(X)$ is the total charge of the measure $\eta$, and $\bar{\eta}=\eta / \eta(X)$ is the normalized process. It follows from the definition of the Lévy process that $\eta(X)$ obeys the infinite divisible law $F_{\Lambda}$ corresponding to the Lévy measure $\Lambda$. As follows from Lemma 1 below and the remark after this lemma, the law of a Lévy process is a product measure in this decomposition if and only if it is a gamma process.

Consider a map $T^{\prime}: D^{+} \rightarrow \mathbb{R}_{+} \times \Sigma \times X^{\infty}$, where

$$
\Sigma=\left\{y=\left(y_{1}, y_{2}, \ldots\right): y_{1} \geq y_{2} \geq \ldots \geq 0, y_{1}+y_{2}+\ldots=1\right\}
$$

is the infinite-dimensional simplex, and

$$
T^{\prime} \eta=\left(\eta(X),\left(Q_{1} / \eta(X), Q_{2} / \eta(X), \ldots\right),\left(X_{1}, X_{2}, \ldots\right)\right)
$$

if $\eta=\sum Q_{i} \delta_{X_{i}}$.

Definition 4. Let $P$ be a probability measure on $D^{+}$, and $\eta=\sum Q_{i} \delta_{X_{i}}$ be a random process obeying $P$. The random normalized sequence of charges $\left(Q_{1} / \eta(X), Q_{2} / \eta(X), \ldots\right)$ is called the simplicial part of the process, and its distribution on $\Sigma$ is called the simplicial part of the law $P$.

3. The gamma process. In this section we summarize basic properties of the gamma processes which we need to prove the Markov-Krein identity $(*)$.

Definition 5. The standard gamma process on the space $(X, \nu)$ is a Lévy process $\gamma$ on $(X, \nu)$ with Lévy measure $d \Lambda(z)=z^{-1} d z, z>0$. Thus the law $\mathcal{G}$ of the gamma process has Laplace transform

$$
\mathbb{E}_{\mathcal{G}}\left[\exp \left(-\int_{X} a(x) d \gamma(x)\right)\right]=\exp \left(-\int_{X} \log (1+a(x)) d \nu(x)\right) .
$$

It is easy to show that formula (4) holds for any measurable function

$$
a \in \mathcal{M}=\left\{a: X \rightarrow \mathbb{R}_{+}: \int_{X} \log (a(x)+1) d \nu(x)<\infty\right\}
$$

Lemma 1, which presents the key property of the gamma process, immediately follows from the corresponding property of gamma variables: if $Y$ and $Z$ are independent gamma variables with the same scale parameter, then the variables $Y+Z$ and $\frac{Y}{Y+Z}$ are independent. 
Lemma 1. The total charge $\gamma(X)$ of the gamma process and the normalized gamma process $\bar{\gamma}=\gamma / \gamma(X)$ are independent. The distribution of the total charge is the gamma distribution with shape parameter $\theta=|\nu|$,

$$
\frac{1}{\Gamma(\theta)} t^{\theta-1} e^{-t} d t, \quad t>0
$$

As follows from a remarkable result of Lukacs [11] for gamma variables, this property is characteristic of the gamma processes. That is, if $\eta$ is a Lévy process such that $\bar{\eta}$ and $\eta(X)$ are independent, then $\eta$ is a gamma process (possibly with some scale parameter).

Lemma 2 ([9]). The simplicial part of the law $\mathcal{G}$ of the gamma process with $|\nu|=\theta$ is the Poisson-Dirichlet measure $P D(\theta)$.

It is easy to see from (3) that for every measurable subset $A \subset X$ the random variable $\gamma(A)$ has gamma distribution with shape parameter $\nu(A)$, and for every measurable partition $A_{0}, \ldots, A_{n}$ of $X$ the variables $\gamma\left(A_{0}\right), \ldots \gamma\left(A_{n}\right)$ are independent. It follows that the vector $\left(\gamma\left(A_{0}\right) / \gamma(X), \ldots, \gamma\left(A_{n}\right) / \gamma(X)\right)$ has Dirichlet distribution on the simplex with parameters $\left(\nu\left(A_{0}\right), \ldots, \nu\left(A_{n}\right)\right)$. Thus the Dirichlet process on the space $X$ with parameter measure $\nu$ is the normalized gamma process on $D(X, \nu)$. One can also establish this relation in terms of the explicit construction (1). Indeed, in view of Theorem 1 and Lemmas 1 and 2, the right-hand side of (1) is the normalized gamma process on $(X, \nu)$.

4. The Markov-Krein identity for means of Dirichlet processes. In this section we prove the MarkovKrein identity for means of Dirichlet processes. It can be interpreted as a formula relating the distribution of a linear functional with respect to the gamma process and the distribution of the same functional with respect to the normalized gamma process. This interpretation allows us to prove it immediately, using only the independence property of the gamma processes.

Given a function $a \in \mathcal{M}$, denote by $\mu_{a}$ the distribution of the linear functional $\eta \mapsto f_{a}(\eta)=\int_{X} a(x) d \eta(x)$ on $D$ with respect to the law $\mathcal{D}$ of the Dirichlet process on $(X, \nu)$ (i.e., the law of the normalized gamma process), and let $\nu_{a}$ be the distribution of the function $a$ with respect to the normalized parameter measure $\bar{\nu}$.

Theorem 2. The measures $\mu_{a}$ and $\nu_{a}$ are related by the following integral identity:

$$
\int_{\mathbb{R}} \frac{1}{(1+z u)^{\theta}} d \mu_{a}(u)=\exp \left(-\int_{X} \log (1+z u)^{\theta} d \nu_{a}(u)\right) .
$$

Note that the left-hand side of (5) is the generalized Cauchy-Stieltjes transform of the distribution $\mu_{a}$. In view of (4), the right-hand side of (5) is equal to the value of the Laplace transform of the gamma process evaluated at the function $a$. Hence one may regard formula (5) as a relation between an integral (CauchyStieltjes) transform of the distribution $\mu_{a}$ of the functional $f_{a}$ with respect to the normalized gamma process and an integral (Laplace) transform of the distribution of the same functional with respect to the nonnormalized gamma process.

Proof. By the Laplace transform formula (4), the right-hand side of (5) equals

$$
\begin{aligned}
& \exp \left(-\int_{X} \log (1+z a(x)) d \nu(x)\right)=\mathbb{E}_{\mathcal{G}}\left[\exp \left(-z \int_{X} a(x) d \gamma(x)\right)\right] \\
& =\mathbb{E}_{\mathcal{G}}\left[\exp \left(-z \gamma(X) \int_{X} a(x) d \bar{\gamma}(x)\right)\right]
\end{aligned}
$$

Since the normalized gamma process and the total charge are independent, the conditional distribution of $\gamma(X)$ given $\bar{\gamma}(\cdot)$ is the same for all $\bar{\gamma}(\cdot)$, and equals the gamma distribution (see Lemma 1 ). Thus we may rewrite the above expression as

$$
\mathbb{E}_{\mathcal{D}}\left[\frac{1}{\Gamma(\theta)} \int_{0}^{\infty} t^{\theta-1} \exp \left(-t-z t \int_{X} a(x) d \bar{\gamma}(x)\right) d t\right] .
$$

Changing the order of integration, we obtain that the last expression equals

and the theorem follows.

$$
\mathbb{E}_{\mathcal{D}}\left[\frac{1}{\left(1+z \int_{X} a(x) d \bar{\gamma}(x)\right)^{\theta}}\right]
$$

A multivariate version of the Markov-Krein identity for the common distributions of several linear functionals of the Dirichlet process was first obtained in [8]. Our proof of Theorem 2 works without essential modifications in the multivariate case too, and gives immediately the desired result. 
Theorem 3. Let $a_{1}, \ldots, a_{n} \in \mathcal{M}$. Denote by $\mu_{a}$ the common distribution of the linear functionals $\left(f_{a_{1}}, \ldots, f_{a_{n}}\right)$ on $D$ with respect to the law $\mathcal{D}$ of the Dirichlet process. Let $\nu_{a}$ be the common distribution of the functions $a_{1}, \ldots, a_{n}$ with respect to the normalized parameter measure $\bar{\nu}$. Then the measures $\mu_{a}$ and $\nu_{a}$ are related by the multivariate Markov-Krein identity

$$
\int_{\mathbb{R}^{n}} \frac{1}{\left(1+z_{1} u_{1}+\ldots+z_{n} u_{n}\right)^{\theta}} d \mu_{a}(u)=\exp \left(-\int_{\mathbb{R}^{n}} \log \left(1+z_{1} u_{1}+\ldots+z_{n} u_{n}\right)^{\theta} d \nu_{a}(u)\right) .
$$

Proof. Goes exactly as the proof of Theorem 2 with function $z a(x)$ replaced by $z_{1} a_{1}(x)+\ldots+z_{n} a_{n}(x)$.

Remark. In fact, Theorem 3 is a particular case of a more general statement. Namely, let $V$ be an arbitrary real topological linear space, and let $\mathcal{L}$ be a space of real linear functionals on $V$. Given a function $a: X \rightarrow V$, we can define a $V$-valued functional $f_{a}(\eta)=\int a(x) d \eta(x)$ on the space $D$ and study the distribution $\mu_{a}$ of this functional with respect to the normalized gamma process. Let $\nu_{a}$ be the distribution of the function $a$ (on the space $V$ ) with respect to $\bar{\nu}$. Then, for an arbitrary linear functional $F \in i \mathcal{L}$, the following formula holds:

$$
\int_{V} \frac{d \mu_{a}(v)}{(1-F(v))^{\theta}}=\exp \int_{V} \log \frac{1}{(1-F(v))^{\theta}} d \nu_{a}(v)
$$

The proof of this formula also reproduces the proof of Theorem 2. Theorem 3 corresponds to a finite-dimensional case: $V=\mathbb{R}^{n}, \mathcal{L}=\left(\mathbb{R}^{n}\right)^{*}$, and $F\left(x_{1}, \ldots, x_{n}\right)=z_{1} x_{1}+\ldots+z_{n} x_{n}$. The Markov-Krein identity in arbitrary linear spaces was first considered in [8, Sect. 9].

5. Two-parameter generalization of the Markov-Krein identity. S. Kerov [7] and J. Pitman [14] independently in different terms suggested the same class of generalizations of the Dirichlet processes. Let $\sigma$ be an arbitrary Borel distribution on the infinite-dimensional simplex $\Sigma$. The generalized Dirichlet process on the space $(X, \nu)$ associated with the distribution $\sigma$ is defined by formula (1), where the sequence $Q$ obeys the law $\sigma$ (instead of $P D(\theta)$ ). An important particular case of the generalized Dirichlet processes is obtained when $\sigma$ is the so-called two-parameter Poisson-Dirichlet distribution $P D(\alpha, \theta)$ [15]. The range of admissible parameters is the union of the sets $\{(\alpha, \theta): \alpha \in(0,1), \theta>-\alpha\}$ and $\{(\alpha,-m \alpha): \alpha<0, m \in \mathbb{N}\}$. If $\alpha=0$, then the measure $P D(0, \theta)$ coincides with the ordinary Poisson-Dirichlet distribution $P D(\theta)$. Denote by $\mathcal{D}(\alpha, \theta)$ the law of the generalized Dirichlet process associated with the two-parameter Poisson-Dirichlet distribution $P D(\alpha, \theta)$.

An analog of the Markov-Krein identity for the distribution of a linear functional with respect to $\mathcal{D}(\alpha, \theta)$ is obtained in [16]. We present here a new proof of this identity which is based on the relation between the two-parameter Poisson-Dirichlet distributions and stable processes.

Definition 6. Let $\alpha \in(0,1)$. The standard $\alpha$-stable process on the space $(X, \nu)$ is a Lévy process with Lévy measure $d \Lambda_{\alpha}=\frac{\alpha}{\Gamma(1-\alpha)} s^{-\alpha-1} d s, s>0$. Thus the law $P_{\alpha}$ of the $\alpha$-stable process has Laplace transform

$$
\mathbb{E}_{P_{\alpha}}\left[\exp \left(-\int_{X} a(x) d \eta(x)\right)\right]=\exp \left(-\int_{X} a(x)^{\alpha} d \nu(x)\right)
$$

where $a: X \rightarrow \mathbb{R}_{+}$is an arbitrary measurable nonnegative function with $\int_{X} a(x)^{\alpha} d \nu(x)<\infty$.

Lemma 3 [15]. The simplicial part of the distribution $P_{\alpha}$ of the $\alpha$-stable process is the generalized PoissonDirichlet distribution $P D(\alpha, 0)$.

The Poisson-Dirichlet distribution $P D(\alpha, \theta)$ with $\alpha, \theta \neq 0$ cannot be represented as the simplicial part of any Lévy process. However, one may obtain it as the simplicial part of a process which has density with respect to a stable process. Namely, let $\theta>-\alpha$, and consider the law $P_{\alpha, \theta}$ on $D$ that has density

$$
\frac{d P_{\alpha, \theta}}{d P_{\alpha}}(\eta)=\frac{c_{\alpha, \theta}}{\eta(X)^{\theta}}
$$

with respect to the $\alpha$-stable law $P_{\alpha}$. Here $c_{\alpha, \theta}=\frac{\Gamma(\theta+1)}{\Gamma(\theta / \alpha+1)}$ is a normalizing constant. 
Lemma 4 [15]. The simplicial part of the measure $P_{\alpha, \theta}$ is the Poisson-Dirichlet distribution $P D(\alpha, \theta)$.

It follows from Lemmas 3 and 4 that the generalized Dirichlet process with parameters $(\alpha, 0)$ is the normalized $\alpha$-stable process, and the generalized Dirichlet process with parameters $(\alpha, \theta)$ is the normalized process governed by the law $P_{\alpha, \theta}$.

Given an arbitrary measurable nonnegative function $a: X \rightarrow \mathbb{R}_{+}$with $\int_{X} a(x)^{\alpha} d \nu(x)<\infty$, let $\mu_{a}$ be the distribution of the functional $f_{a}$ with respect to $\mathcal{D}(\alpha, \theta)$, and let $\nu_{a}$ be the distribution of $a$ with respect to the normalized parameter measure $\bar{\nu}$.

Theorem 4. The measures $\mu_{a}$ and $\nu_{a}$ are related by the following integral identity:

(1) if $\theta \neq 0$, then

$$
\left(\int_{\mathbb{R}}(1+z u)^{-\theta} d \mu_{a}(u)\right)^{-\frac{1}{\theta}}=\left(\int_{\mathbb{R}}(1+z u)^{\alpha} d \nu_{a}(u)\right)^{\frac{1}{\alpha}}
$$

(2) if $\theta=0$, then

$$
\exp \left(\int_{\mathbb{R}} \log (1+z u)^{\alpha} d \mu_{a}(u)\right)=\int_{\mathbb{R}}(1+z u)^{\alpha} d \nu_{a}(u) .
$$

Proof. (1) Denote the left-hand side of the desired identity by $A^{-1 / \theta}$, and the right-hand side by $B^{1 / \alpha}$. Using the identity

$$
\frac{1}{r^{\theta}}=\frac{1}{\Gamma(\theta)} \int_{0}^{\infty} t^{\theta-1} e^{-r t} d t
$$

we obtain

$$
\begin{aligned}
A & =c_{\alpha, \theta} \mathbb{E}^{\alpha}\left[\left(\eta(X)+z \int_{X} a(x) d \eta(x)\right)^{-\theta}\right] \\
& =\frac{c_{\alpha, \theta}}{\Gamma(\theta)} \mathbb{E}^{\alpha}\left[\int_{0}^{\infty} t^{\theta-1} \exp \left(-t\left(\eta(X)+z \int_{X} a(x) d \eta(x)\right)\right) d t\right] \\
& =\frac{c_{\alpha, \theta}}{\Gamma(\theta)} \int_{0}^{\infty} t^{\theta-1} \mathbb{E}^{\alpha}\left[\exp \left(-\int_{X} t(1+z a(x)) d \eta(x)\right)\right] d t
\end{aligned}
$$

By the Laplace transform formula (7), the expectation equals precisely $e^{-t^{\alpha} B}$, thus

$$
A=\frac{c_{\alpha, \theta}}{\Gamma(\theta)} \int_{0}^{\infty} t^{\theta-1} e^{-t^{\alpha} B} d t
$$

and (9) follows by changing variables.

(2) Follows from (9) by letting $\theta \rightarrow 0$.

The first and the third authors are partially supported by RFBR grant 99-01-00098, the first author is also supported by CRDF grant RM1-2244.

Added in the translation. The identity involving the gamma function which we use in the proof of Theorem 4 makes sense only for positive $\theta$. The authors are grateful to L. James for this remark. In fact, for $-\alpha<\theta<0$ one should use instead the relation $\Gamma(\theta)=\frac{\Gamma(\theta+1)}{\theta}$ and the identity for $\theta+1$ which is already positive.

Translated by N. V. Tsilevich.

\section{REFERENCES}

1. A.Borodin and G.Olshanski, "Distributions on partitions, point processes and the hypergeometric kernel," Comm. Math. Phys., 211, No. 2, 335-358 (2000).

2. D. M. Cifarelli and E. Regazzini, "Some remarks on the distribution functions of means of a Dirichlet process," Ann. Statist., 18, 429-442 (1990).

3. P. Diaconis and J. Kemperman, "Some New Tools for Dirichlet Priors," in: Bayesian Statistics 5, J. M. Bernardino, J. O. Berger, A. P. Dawid and A. F. M. Smith (eds.), Oxford University Press (1996), pp. 97-106. 
4. T. S. Ferguson, "A Bayesian analysis of some nonparametric problems," Ann. Statist., 1, $209-230$ (1973).

5. T. S. Ferguson and M. J. Klass, "A representation of independent increment processes without Gaussian components," Ann. Math. Statist., 43, 1634-1643 (1972).

6. S. V. Kerov, "Transition probabilities of continuous Young diagrams and Markov moment problem," Funct. Anal. Appl., 27, 32-49 (1993).

7. S. V. Kerov, "Interlacing Measures," Amer. Math. Soc. Transl. (2), 181, 35-83 (1998).

8. S. V. Kerov and N. V. Tsilevich, "The Markov-Krein correspondence in several dimensions," PDMI Preprint $1 / 1998$.

9. J. F. C. Kingman, "Random discrete distributions," J. Roy. Statist. Soc. B, 37, 1-22 (1975).

10. M. G. Krein and A. A. Nudelman, The Markov Moment Problem and Extremal Problems [in Russian], Nauka, Moscow (1973).

11. E. Lukacs, "A characterization of the gamma distribution," Annals Math. Stat., 26, 319-324 (1965).

12. I. G. Macdonald, Symmetric Functions and Hall Polynomials, Clarendon Press, Oxford (1979).

13. A. A. Markov, "Nouvelles applications des fractions continues," Math. Ann., 47, 579-597 (1896).

14. J. Pitman, "Some developments of the Blackwell-MacQueen urn scheme," in: Statistics, Probability and Game theory, T. S. Ferguson, L. S. Shapley, J. B. MacQueen (eds.), IMS Lecture Notes - Monograph series, 30 (1996), pp. 245-267.

15. J. Pitman and M. Yor, "The two-parameter Poisson-Dirichlet distribution derived from a stable subordinator," Ann. Prob., 25, 855-900 (1997).

16. N. V. Tsilevich, "Distributions of mean values for some random measures," Zap. Nauchn. Semin. POMI, 240, 268-279 (1997).

17. N. V. Tsilevich and A. M. Vershik, "Quasi-invariance of the gamma process and multiplicative properties of the Poisson-Dirichlet measures," C. R. Acad. Sci. Paris, Série I, 329, No. 2, 163-168 (1999).

18. N. V. Tsilevich, A. M. Vershik, and M.Yor, "Distinguished properties of the gamma process and related topics," Prépublications du Laboratoire de Probabilités et Modèles aléatoires, No. 575 (2000).

19. N. V. Tsilevich, A. M. Vershik, and M.Yor, "An infinite-dimensional analogue of the Lebesgue measure and distinguished properties of the gamma process," J. Funct. Anal, 185, No. 1, 274-296 (2001). 